\title{
Impact of beta-blockers on prostate cancer mortality: a meta-analysis of 16,825 patients
}

OncoTargets and Therapy

30 April 2015

Number of times this article has been viewed

\author{
Hua Lu ${ }^{1,2}$ \\ Xingjie Liu $^{2}$ \\ Fengfu Guo ${ }^{2}$ \\ Shanfeng Tan ${ }^{2}$ \\ Guangjian Wang² \\ Hongjun Liu ${ }^{2}$ \\ Jianming Wang ${ }^{2}$ \\ Xiangfei $\mathrm{He}^{2}$ \\ Yanshuai $\mathrm{Mo}^{2}$ \\ Benkang Shi \\ 'Department of Urology, Qilu \\ Hospital of Shandong University, \\ Shandong, People's Republic of \\ China; ${ }^{2}$ Department of Urology, Linyi \\ People's Hospital, Shandong, People's \\ Republic of China
}

Introduction: Increasing evidence suggests that beta-blocker use might be associated with reduced mortality in prostate cancer patients. To provide a quantitative assessment of this association, we pooled data available to examine the association between beta-blocker use and mortality of prostate cancer.

Methods: We identified studies by a literature search of MEDLINE (from 1 January 1966) and EMBASE (from 1 January 1974), through 10 September 2014, and by searching the reference lists of pertinent articles. Two authors independently screened and reviewed the eligibility of each study. The primary outcomes were prostate cancer-specific mortality and all-cause mortality. Results: A total of four studies including 16,825 patients were included in this meta-analysis. Analysis of all studies showed that beta-blocker use was associated with reduced prostate cancer-specific mortality (hazard ratio $=0.85,95 \%$ confidence interval $=0.77-0.94$ ), without any heterogeneity between studies $\left(Q=3.59, I^{2}=16.5 \%, P=0.309\right)$. However, we observed no association with all-cause mortality (hazard ratio $=0.97,95 \%$ confidence interval $=0.90-1.04$ ). There was also no evidence of the presence of significant heterogeneity between the four studies $\left(Q=2.48, I^{2}=0.0 \%, P=0.480\right)$.

Conclusion: These findings indicate that beta-blocker use was associated with reduced cancerspecific mortality among prostate cancer patients taking beta-blockers.

Keywords: prostate cancer, beta-blocker, mortality, meta-analysis

\section{Introduction}

Prostate cancer is one of the most common malignancies of the urinary tract. Based on incidence and mortality data from several agencies, the American Cancer Society estimates that 233,000 new prostate cancer cases and 29,480 deaths from prostate cancer are projected to occur in the United States in 2014. ${ }^{1}$ Since 1998, the incidence rate for prostate cancer has generally increased. ${ }^{2}$ Moreover, the mortality rate of prostate cancer has also dramatically increased in recent years. ${ }^{3}$ Prostate cancer affects elderly men more often and therefore is a bigger health concern in developed countries. Approximately $15 \%$ of male cancers is prostate cancer in developed countries, compared with $4 \%$ of male cancers in developing countries. ${ }^{4}$

Beta-adrenergic signaling has been found to regulate multiple cellular processes that contribute to the initiation and progression of cancer. ${ }^{5}$ In animal studies, it has been demonstrated that beta-blockers could inhibit the development of metastases from breast cancer, ${ }^{6,7}$ and prevent stress-induced tumor growth and angiogenesis in ovarian carcinoma models. ${ }^{8}$ Palm et al also found that the beta-blocker propranolol inhibited the development of lumbar lymph node metastases in athymic BALB/c nude mice injected with human prostate cancer cells. ${ }^{9}$

The potential of beta-blockers to inhibit cancer progression has received increasing interest. Beta-blockers were also found to increase the anti-angiogenic effects 
and anti-tumor efficacy of certain chemotherapy drugs both in vitro and in vivo, in several mouse models of human cancer. ${ }^{10-12}$ Preclinical pharmacologic and biomarker studies are now laying the groundwork for translation of beta-blockade as a novel adjuvant to existing therapeutic strategies in clinical oncology. Epidemiological studies have also shown that beta-blockers might be associated with longer survival and reduced mortality in patients with non-small-cell lung cancer, colorectal cancer, melanoma, and prostate cancer. ${ }^{13-16}$

Despite these promising studies, data on the effect of beta-blocker usage on prostate cancer-specific mortality and all-cause mortality are limited and inconsistent. ${ }^{17-20}$ Cardwell et al found that beta-blocker usage after diagnosis was not associated with cancer-specific or all-cause mortality in prostate cancer patients in a large UK study. ${ }^{19}$ Grytli et al reported that beta-blocker use was associated with reduced prostate cancer-specific mortality in patients with high-risk or metastatic disease at the time of diagnosis. ${ }^{20}$ Accordingly, we performed a meta-analysis to pool studies currently available to examine the effect of beta-blocker use on cancer mortality among patients with prostate cancer.

\section{Patients and methods}

This systematic review and meta-analysis was performed according to the recently published recommendations and checklist of the Preferred Reporting Items for Systematic Reviews and Meta-Analyses statement and the Preferred Reporting Items for Meta-Analysis of Observational Studies in Epidemiology (MOOSE) guidelines. ${ }^{21}$

\section{Data sources and searches}

A computerized literature search was conducted using MEDLINE (from 1 January 1966) and EMBASE (from 1 January 1974) through 10 June 2014 by two independent investigators. The search strategy used medical subject heading (MeSH) terms and keywords: beta-blocker; prostate; neoplasm(s) or cancer; mortality; survival; mortality; atenolol; metoprolol; propranolol; betaxolol; bevantolol; carteolol; bisoprolol; carvedilol; celiprolol; arotinolol; and epidemiologic studies. We also manually reviewed the reference lists to identify additional relevant studies. No language restrictions were imposed.

\section{Study selection}

We included those studies that met all of the following criteria: 1) participants were diagnosed with prostate cancer; 2) the exposure of interest was beta-blocker; 3) outcome variable must be the survival time; 4) studies must be comparative. Articles or reports from non-peer-reviewed sources were not included in this meta-analysis.

A study was excluded from this meta-analysis under the following conditions: 1) if multi-center studies contained data which were already included in a single center study; and 2 ) if a hazard ratio (HR) with $95 \%$ confidence interval (CI) for survival was not directly reported, or it was impossible to calculate that from the paper. One study was excluded because it presented results on survival by cancer site for patients receiving any regular beta-adrenoceptor blocker therapy compared with patients receiving other blood pressure-lowering medication. ${ }^{17}$

\section{Data extraction}

Data were extracted independently by two authors and crosschecked to reach a consensus. When discrepancies were found, a third investigator would make the definitive decision for study eligibility and data extraction. The following variables were recorded: the first author's last name, publication year, geographic region of original study, sample size, participant characteristics, length of follow-up, variables adjusted in the analysis, and the HRs with corresponding $95 \%$ CIs. In order to study the difference in survival of prostate cancer patients receiving beta-blocker or not, we pooled the HRs of prostate cancer-specific mortality and all-cause mortality. From each study, we extracted the HR estimate that was adjusted for the greatest number of potential confounders.

\section{Statistical analysis}

Our primary analyses were focused on a comparison of the HR of mortality of prostate cancer patients. In cases in which multiple statistical tests were used to assess an association, results of the most rigorous test were reported (eg, a multivariable analysis was preferred over a univariable analysis). All statistical analyses were performed using STATA version 11.0 (StataCorp LP, College Station, TX, USA). A two-tailed $P$-value of less than 0.05 was considered to be statistically significant. The variance of the $\log \mathrm{HR}$ from each study was calculated by converting the $95 \%$ CI to its natural logarithm by taking the width of the CI and dividing by 3.92 . Summary HR estimates with corresponding 95\% CIs were derived using the method of DerSimonian and Laird ${ }^{22}$ with the assumptions of a random-effects model, which considers both within-study and between-study variations. In assessing heterogeneity among studies, we used the Cochran $Q$ and $I^{2}$ statistics. ${ }^{23}$ These were used to test whether the differences 


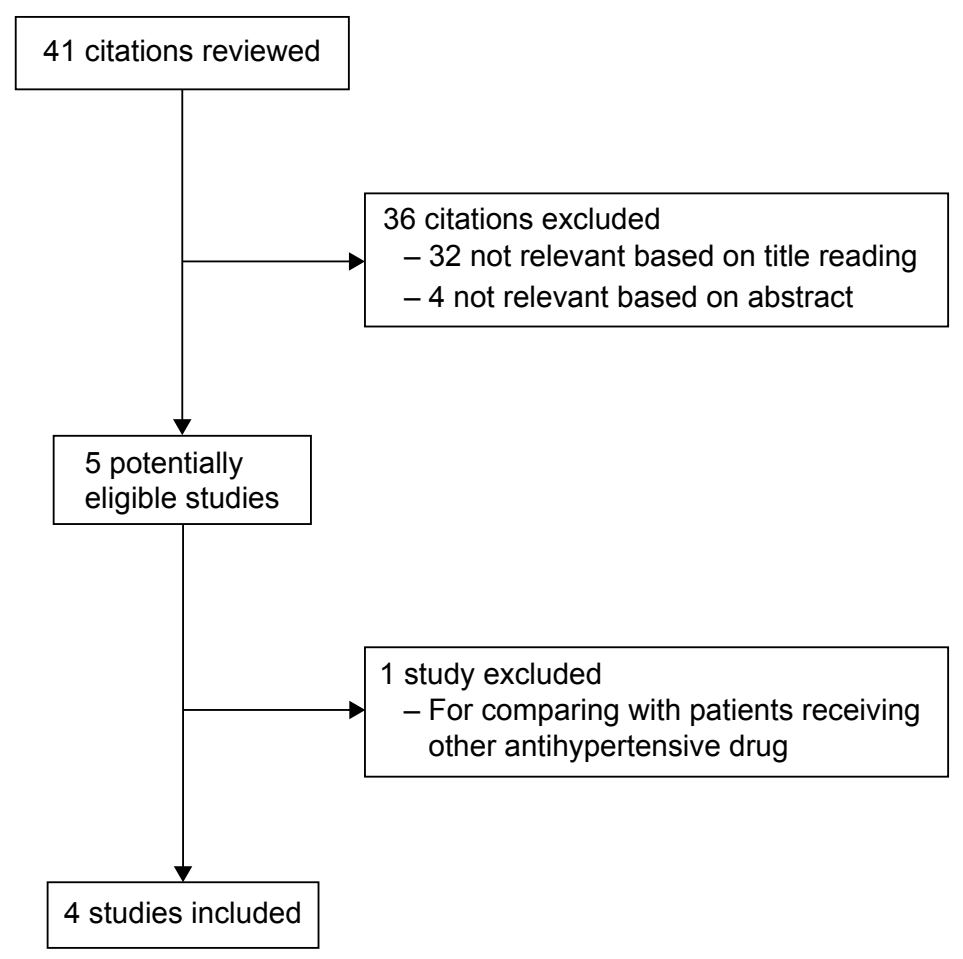

Figure I Flow chart on the article selection process.

obtained between studies were due to chance. For the $Q$ statistic, a $P$-value of less than 0.10 was used as an indication of the presence of heterogeneity; for $I^{2}$, a value $>50 \%$ was considered a measure of severe heterogeneity.

\section{Results}

All published studies analyzing the effect of beta-blockers on mortality of prostate cancer patients were included if they met the inclusion criteria. The details of the literature search are presented in a flow diagram (Figure 1). A total of 41 citations were identified from the MEDLINE and EMBASE database. We excluded 36 citations after screening based on titles or abstracts. After this, five remaining citations were full-text reviewed. In one of the studies, beta-blocker use was compared with other antihypertensive medication, and thus, we had to exclude this study due to improper treatment and control groups. ${ }^{17}$
Finally, four citations including 16,825 patients met the inclusion criteria and were included in our meta-analysis. ${ }^{18-20,24}$ The characteristics of the included studies are shown in Table 1. One study investigated the association of betablocker use with survival in prostate cancer patients on androgen deprivation therapy. ${ }^{18}$ One study assessed the association between beta-blockers and prostate cancerspecific mortality in prostate cancer patients with high-risk or metastatic disease. ${ }^{20}$ The third study investigated the effect of beta-blockers on prostate cancer-specific mortality in a large UK study. ${ }^{19}$ The fourth study determined whether post-diagnostic use of beta-blockers was associated with prostate cancer mortality and all-cause mortality. ${ }^{24}$ Two original studies included prostate cancer patients only, ${ }^{20,24}$ and the other two studies also had cancer-free men. ${ }^{18,19}$ Three studies ${ }^{19,20,24}$ analyzed beta-blocker usage after prostate cancer diagnosis, and the other one ${ }^{18}$ analyzed beta-blocker usage

Table I The characteristics of included studies for meta-analysis

\begin{tabular}{|c|c|c|c|c|c|c|c|c|}
\hline \multirow[t]{2}{*}{ Study } & \multirow[t]{2}{*}{ Year } & \multirow[t]{2}{*}{ Country } & \multirow[t]{2}{*}{ No of patients } & \multirow{2}{*}{$\begin{array}{l}\text { Mean age, } \\
\text { years }\end{array}$} & \multicolumn{2}{|c|}{ Beta blocker use } & \multirow{2}{*}{$\begin{array}{l}\text { Follow-up, } \\
\text { months }\end{array}$} & \multirow[t]{2}{*}{ End point } \\
\hline & & & & & Yes & No & & \\
\hline Grytli et al ${ }^{18}$ & 2013 & Norway & 655 & 72.1 & 80 & 575 & 122 & CSM, ACM \\
\hline Grytli et a ${ }^{20}$ & 2014 & Norway & 3,561 & 76.3 & 1,115 & 2,446 & 39 & CSM, ACM \\
\hline Cardwell et al ${ }^{19}$ & 2014 & UK & 6,339 & 1 & 1,225 & 3,490 & 72 & CSM, ACM \\
\hline Assayag et $\mathrm{a}^{24}$ & 2014 & UK & 6,270 & 72.3 & 673 & 1,088 & 45.6 & CSM, ACM \\
\hline
\end{tabular}

Abbreviations: CSM, cancer-specific mortality; ACM, all-cause mortality. 
Study

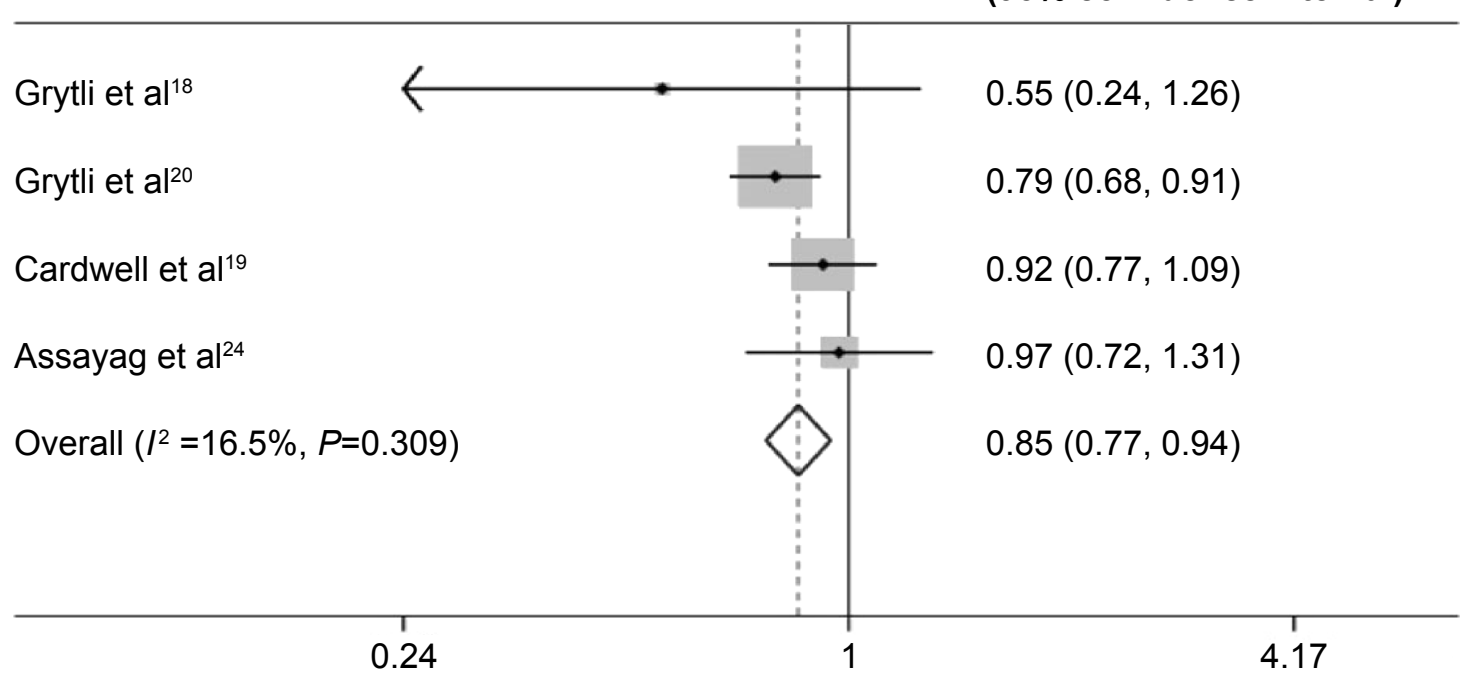

Hazard ratio

(95\% confidence interval)

Figure 2 Forest plot of prostate cancer-specific mortality associated with beta-blockers. Note: 0.24 to 4.17 reflects the maximal range of Hazard ratio.

both before and after prostate cancer diagnosis. Our results showed that beta-blocker use was associated with reduced prostate cancer-specific mortality (HR 0.85 ; $95 \%$ CI, 0.77 $0.94)$, without any heterogeneity between studies $(Q=3.59$, $I^{2}=16.5 \%, P=0.309$ ) (Figure 2). However, we observed no association with all-cause mortality (HR $0.97 ; 95 \% \mathrm{CI}$, $0.90-1.04)$. There was also no evidence of the presence of significant heterogeneity between the four studies $(Q=2.48$, $P=0.480 ; I^{2}=0.0 \%$ ) (Figure 3 ).

\section{Discussion}

Previous in vitro data suggest that beta-blockers exert an antitumor effect on cancer cell lines, and stimulation of the sympathetic nervous system induces metastases in xenograft models of breast, ovarian, and prostate cancer. ${ }^{6-9,25}$ The main mediator of these effects is thought to be ADRB2. ${ }^{7,8}$ The signaling pathways of the ADRBs have also been suggested to be involved in the immune system's response to cancer, in addition to having effects on angiogenesis and cancer cell

\section{Study}

Hazard ratio

(95\% confidence interval)

\begin{tabular}{|c|c|}
\hline Grytli et al ${ }^{18}$ & $0.88(0.56,1.38)$ \\
\hline Grytli et al ${ }^{20}$ & $0.92(0.83,1.02)$ \\
\hline Cardwell et al ${ }^{19}$ & $1.04(0.92,1.17)$ \\
\hline Assayag et $\mathrm{al}^{24}$ & $0.97(0.81,1.16)$ \\
\hline Overall $\left(I^{2}=0.0 \%, P=0.480\right)$ & $0.97(0.90,1.04)$ \\
\hline 0.56 & 1.78 \\
\hline
\end{tabular}

Figure 3 Forest plot of prostate cancer all-cause mortality associated with beta-blockers. 
migration. Thus, Benish et al stated that treatment combining perioperative beta-blockade might be practical in operated cancer patients, and their study suggested potential immunological and clinical benefits. ${ }^{26}$

Kim-Fuchs et al demonstrated that pharmacological activation of beta-adrenergic signaling induced similar effects to chronic stress, and pharmacological beta-blockade reversed the effects of chronic stress on pancreatic cancer progression. ${ }^{27}$ Hassan et al also reported that stress promoted prostate carcinogenesis in mice in an adrenaline-dependent manner. Of note, the effects of stress could be prevented by treatment with a selective ADRB2 antagonist. ${ }^{28}$ Perron et al demonstrated that beta-blockers were associated with a reduction in prostate cancer risk, ${ }^{29}$ whereas Kemppainen et al reported increased prostate cancer risk among men using beta-blockers. ${ }^{30}$ Noteworthy, the association between beta-blocker use and survival of prostate cancer patients remains unclear.

Shah et al observed that patients receiving beta-blocker therapy experienced slightly poorer survival, and the overall higher mortality in patients receiving beta-blockers was explained by patients with pancreatic and prostate cancer. ${ }^{17}$ Considering that beta-blocker was compared with other antihypertensive medication, further study is needed to clarify this association. Since the study was published, a variety of relevant studies on this association have yielded inconsistent results. Grytli et al demonstrated a possible benefit of beta-blocker use for prostate cancer patients treated with androgen deprivation therapy. ${ }^{18}$ However, beta-blocker usage after diagnosis was not associated with cancer-specific or all-cause mortality in prostate cancer patients in a large UK study. ${ }^{19}$

Currently, we analyzed this relationship further by conducting a meta-analysis of four studies including 16,825 prostate cancer patients. We reported reduced cancer-specific mortality for beta-blocker users in prostate cancer patients. However, we found no evidence of a reduction in all-cause mortality in beta-blocker users in our analysis. Pasquier et al observed that propranolol could potentiate the antiangiogenic effects and antitumor efficacy of chemotherapy in breast cancer. ${ }^{12}$ They also found that beta-blockers could increase treatment efficacy against neuroblastoma, and their combination with chemotherapy might prove beneficial for the treatment of this disease and other drug-refractory cancers. ${ }^{10}$ Taxanes are often used in the treatment of prostate cancer, and their synergistic interactions with beta-blockers could result in improved outcome for prostate cancer patients. ${ }^{31,32}$ Thus, the interaction with chemotherapy may be critical in the observed survival benefits of prostate cancer patients.
Considering the increasing incidence of prostate cancer, our results have important clinical and public health significance. Our literature search was extensive; we tested for and found no evidence of publication bias. The strength of the study is that, on an international scale, there are far more individuals with prostate cancer in our study.

Our study also has several potential limitations of the available data. Thus, caution is needed when interpreting these results. First, different study designs may have particular methodological issues and constraints. Casecontrol studies are susceptible to recall and selection biases which could inflate the HRs. Cohort studies are prone to be influenced by detection bias because prostate patients receiving beta-blockers are under increased medical surveillance.

Second, we could not analyze the type of beta-blocker that produced these effects because most studies combined beta-blockers in their analyses. Third, we could not compare how the study population, such as patients with early-/latestage cancer or patients treated with/without surgery, influence the effect size. Finally, inherent in any meta-analysis of published data is the possibility of publication bias, that is small studies with null results tend not to be published. However, the results obtained from funnel plot analysis and formal statistical tests (data not shown) did not provide evidence for such bias.

\section{Conclusion}

In summary, though there are some limitations in this study, we observed reduced cancer-specific mortality among prostate cancer patients taking beta-blockers. However, we did not observe any effect of beta-blocker use on all-cause mortality in this meta-analysis. Taken together with studies in other cancer types and in preclinical models, our findings indicate a beneficial effect of beta-blockers on survival in patients with prostate cancer. Therefore, beta-blockers may be considered a promising therapeutic approach for adjuvant therapy in prostate cancer. Further clinical trials must be explored in larger patient cohorts.

\section{Disclosure}

The authors declare that they have no conflict of interest.

\section{References}

1. Siegel R, Ma J, Zou Z, Jemal A. Cancer statistics, 2014. CA Cancer J Clin. 2014;64(1):9-29.

2. Han S, Zhang S, Chen W, et al. [Analysis of the status and trends of prostate cancer incidence in China]. Chin Clin Oncol. 2013;(04):330-334. Chinese. 
3. Han S, Zhang S, Chen W, et al. [Analysis of the status and trends of prostate cancer mortality in China]. Chin J Urol. 2012;33(11):836-839. Chinese.

4. Heidenreich A, Bastian PJ, Bellmunt J, et al. EAU guidelines on prostate cancer. part 1: screening, diagnosis, and local treatment with curative intent-update 2013. Eur Urol. 2014;65(1):124-137.

5. Cole SW, Sood AK. Molecular pathways: beta-adrenergic signaling in cancer. Clin Cancer Res. 2012;18(5):1201-1206.

6. Sloan EK, Priceman SJ, Cox BF, et al. The sympathetic nervous system induces a metastatic switch in primary breast cancer. Cancer Res. 2010;70(18):7042-7052.

7. Campbell JP, Karolak MR, Ma Y, et al. Stimulation of host bone marrow stromal cells by sympathetic nerves promotes breast cancer bone metastasis in mice. PLoS Biol. 2012;10(7):e1001363.

8. Thaker PH, Han LY, Kamat AA, et al. Chronic stress promotes tumor growth and angiogenesis in a mouse model of ovarian carcinoma. Nat Med. 2006;12(8):939-944.

9. Palm D, Lang K, Niggemann B, et al. The norepinephrine-driven metastasis development of PC-3 human prostate cancer cells in BALB/c nude mice is inhibited by beta-blockers. Int J Cancer. 2006;118(11):2744-2749.

10. Pasquier E, Street J, Pouchy C, et al. beta-blockers increase response to chemotherapy via direct antitumour and anti-angiogenic mechanisms in neuroblastoma. Br J Cancer. 2013;108(12):2485-2494.

11. Wolter JK, Wolter NE, Blanch A, et al. Anti-tumor activity of the beta-adrenergic receptor antagonist propranolol in neuroblastoma. Oncotarget. 2014;5(1):161-172.

12. Pasquier E, Ciccolini J, Carre M, et al. Propranolol potentiates the anti-angiogenic effects and anti-tumor efficacy of chemotherapy agents: implication in breast cancer treatment. Oncotarget. 2011;2(10): 797-809.

13. Botteri E, Munzone E, Rotmensz N, et al. Therapeutic effect of betablockers in triple-negative breast cancer postmenopausal women. Breast Cancer Res Treat. 2013;140(3):567-575.

14. De Giorgi V, Gandini S, Grazzini M, Benemei S, Marchionni N, Geppetti P. Effect of beta-blockers and other antihypertensive drugs on the risk of melanoma recurrence and death. Mayo Clin Proc. 2013;88(11): 1196-1203.

15. Wang HM, Liao ZX, Komaki R, et al. Improved survival outcomes with the incidental use of beta-blockers among patients with non-small-cell lung cancer treated with definitive radiation therapy. Ann Oncol. 2013; 24(5):1312-1319.

16. Jansen L, Hoffmeister M, Arndt V, Chang-Claude J, Brenner H. Stagespecific associations between beta blocker use and prognosis after colorectal cancer. Cancer. 2014;120(8):1178-1186.

17. Shah SM, Carey IM, Owen CG, Harris T, Dewilde S, Cook DG. Does beta-adrenoceptor blocker therapy improve cancer survival? Findings from a population-based retrospective cohort study. Br J Clin Pharmacol. 2011;72(1):157-161.
18. Grytli HH, Fagerland MW, Fossa SD, Tasken KA, Haheim LL. Use of beta-blockers is associated with prostate cancer-specific survival in prostate cancer patients on androgen deprivation therapy. Prostate. 2013;73(3):250-260.

19. Cardwell CR, Coleman HG, Murray LJ, O’Sullivan JM, Powe DG. Beta-blocker usage and prostate cancer survival: A nested case-control study in the UK Clinical Practice Research Datalink cohort. Cancer Epidemiol. 2014;38(3):279-285.

20. Grytli HH, Fagerland MW, Fossa SD, Tasken KA. Association between use of beta-blockers and prostate cancer-specific survival: a cohort study of 3,561 prostate cancer patients with high-risk or metastatic disease. Eur Urol. 2014;65(3):635-641.

21. Stroup DF, Berlin JA, Morton SC, et al. Meta-analysis of observational studies in epidemiology: a proposal for reporting. Meta-analysis Of Observational Studies in Epidemiology (MOOSE) group. JAMA. 2000; 283(15):2008-2012.

22. DerSimonian R, Laird N. Meta-analysis in clinical trials. Control Clin Trials. 1986;7(3):177-188.

23. Higgins JP, Thompson SG. Quantifying heterogeneity in a metaanalysis. Stat Med. 2002;21(11):1539-1558.

24. Assayag J, Pollak MN, Azoulay L. Post-diagnostic use of beta-blockers and the risk of death in patients with prostate cancer. Eur J Cancer. 2014; 50(16):2838-2845

25. Lutgendorf SK, Cole S, Costanzo E, et al. Stress-related mediators stimulate vascular endothelial growth factor secretion by two ovarian cancer cell lines. Clin Cancer Res. 2003;9(12):4514-4521.

26. Benish M, Bartal I, Goldfarb Y, et al. Perioperative use of beta-blockers and COX-2 inhibitors may improve immune competence and reduce the risk of tumor metastasis. Ann Surg Oncol. 2008;15(7):2042-2052.

27. Kim-Fuchs C, Le CP, Pimentel MA, et al. Chronic stress accelerates pancreatic cancer growth and invasion: A critical role for beta-adrenergic signaling in the pancreatic microenvironment. Brain Behav Immun. 2014;40:40-47.

28. Hassan S, Karpova Y, Baiz D, et al. Behavioral stress accelerates prostate cancer development in mice. J Clin Invest. 2013;123(2):874-886.

29. Perron L, Bairati I, Harel F, Meyer F. Antihypertensive drug use and the risk of prostate cancer (Canada). Cancer Causes Control. 2004; 15(6):535-541

30. Kemppainen KJ, Tammela TL, Auvinen A, Murtola TJ. The association between antihypertensive drug use and incidence of prostate cancer in Finland: a population-based case-control study. Cancer Causes Control. 2011;22(10):1445-1452.

31. Mita AC, Figlin R, Mita MM. Cabazitaxel: more than a new taxane for metastatic castrate-resistant prostate cancer. Clin Cancer Res. 2012; 18(24):6574-6579.

32. Schutz FA, Buzaid AC, Sartor O. Taxanes in the management of metastatic castration-resistant prostate cancer: efficacy and management of toxicity. Crit Rev Oncol Hematol. 2014;91(3):248-256.
OncoTargets and Therapy

\section{Publish your work in this journal}

OncoTargets and Therapy is an international, peer-reviewed, open access journal focusing on the pathological basis of all cancers, potential targets for therapy and treatment protocols employed to improve the management of cancer patients. The journal also focuses on the impact of management programs and new therapeutic agents and protocols on
Dovepress

patient perspectives such as quality of life, adherence and satisfaction The manuscript management system is completely online and includes a very quick and fair peer-review system, which is all easy to use. Visit http://www.dovepress.com/testimonials.php to read real quotes from published authors. 